\title{
Identification of Lead Compounds for Inhibitor Design against Tyrosyl DNA Phosophodiesterase I by Crystallographic Fragment Screening
}

\author{
George T. Lountos ${ }^{1,4}$, Xue Zhi Zhao ${ }^{2}$, Evgeny Kiselev ${ }^{3}$, Joseph E. Tropea ${ }^{4}$, Danielle Needle ${ }^{4}$, \\ Terrence R. Burke, Jr. ${ }^{2}$, Yves Pommier ${ }^{3}$, and David S. Waugh ${ }^{4}$ \\ ${ }^{1}$ Basic Science Program, Leidos Biomedical Research, Inc. Frederick, National Laboratory for \\ Cancer Research, ${ }^{2}$ Chemical Biology Laboratory, $\mathrm{NCl}$ at Frederick, ${ }^{3}$ Developmental Therapeutics \\ Branch, National Cancer Institute, ${ }^{4}$ Macromolecular Crystallography Laboratory, $\mathrm{NCl}$ at \\ Frederick
}

Fragment-based inhibitor discovery is a powerful approach that can be applied to identify lead compounds for challenging protein targets in drug discovery efforts. Fragments used in screening libraries are typically small compounds ( $<250$ Daltons) that exhibit favorable drug like properties but typically bind very weakly to the target. In order to identify such weak binders, sensitive techniques such as X-ray crystallography, NMR, or surface plasmon resonance must be utilized. Despite their weak binding, fragments identified through fragment-based methods can provide excellent starting points for the development of highly potent and specific inhibitors even for very challenging targets that are often classified as "undruggable". Screening by X-ray crystallography can confirm the binding of a fragment to the target and concurrently offers the precise structural information needed to elaborate the fragment into a viable inhibitor. TyrosylDNA phosphodiesterase I (TDP1) is a critical enzyme that repairs topoisomerase (TOP1) cleavage complexes generated by inhibitors of TOP1 and blocked replication intermediates generated by cytarabine, acyclovir and zidovudine. TDP1 also removes oxidative products from the 3 '-end of DNA breaks both in the nuclear and mitochondrial genomes. It is hypothesized that combination chemotherapy with TDP1 inhibitors should synergize with topoisomerase targeting drugs to enhance selectivity against cancer cells exhibiting deficiencies in parallel DNA repair pathways. As a first step towards TDP1 inhibitor development, we have initiated a crystallographic screening campaign using a fragment library of 640 fragments against TDP1. Crystal structures, solved up to $1.6 \AA$ resolution, identified three fragments that bind to the TDP1 active site and biochemical assays confirm that the fragments exhibit inhibitory activity against TDP1. Using structural insights into fragment binding, several derivatives with increased potency have been designed, prepared and evaluated in biochemical assays. These results demonstrate that fragment-based methods can be a highly beneficial approach towards the discovery of TDP1 inhibitors and provide the first co-crystal structures of small molecule inhibitors bound to TDP1. 